\section{Kurativ behandeltes Osteosarkom: Prognose- Nomogramm hilft bei weiterer Beratung}

\author{
Nomogramme sind bei Tumorerkrankungen zum individuellen Risiko- \\ management eine wichtige Hilfe. Nun gibt es auch ein Nomogramm zur \\ Abschätzung des Metastasierungsrisikos beim kurativ therapierten \\ Osteosarkom auf Basis eines größeren Patientenkollektivs.
}

D as Osteosarkom ist eine seltene Entität. Bisher gab es deshalb nur 2 Nomogramme mit begrenzter Aussagekraft. Ein japanischer Arbeitskreis hat nun anhand einer vergleichsweise großen Patientenzahl ein Nomogramm zur Vorhersage des metastasenfreien Überlebens (MFS) sowie des Gesamtüberlebens (OS) für Patienten nach kurativer neoadjuvanter Chemotherapie und Operation entwickelt und extern validiert.

Einbezogen in das Projekt wurden 1.070 Patienten. 557 von ihnen waren in japanischen Institutionen behandelt worden, ihre Daten dienten retrospektiv der Entwicklung des Nomogramms mit interner Validierung per Bootstrap-
Resampling. Danach erfolgte die externe Validierung anhand der retrospektiven Daten einer unabhängigen Patientenkohorte $(\mathrm{n}=513)$ aus Korea.

Insgesamt haben die drei Variablen Patientenalter, Tumorlokalisation und histologisches Ansprechen einen größeren Einfluss auf die beiden Endpunkte als die Variablen Geschlecht, Tumorgröße und Frakturen. Die Vorhersagewahrscheinlichkeit für die Endpunkte MFS bzw. OS in der internen Validation mit Konkordanzindizes von 0,631 bzw. 0,679 war relativ gut. In der externen Validation betrug der Konkordanzwert für das MFS 0,682 und für das OS 0,665. Mithilfe dieses 6-Variablen-Nomogramms sollte es für die behandelnden Ärzte in Zukunft leichter sein, den besten Zeitpunkt für die Nachbeobachtungstermine festzulegen und auf diese Weise überflüssige Untersuchungen zu vermeiden, ohne eine mögliche Metastasenentwicklung zu verpassen. Limitiert werde die Tauglichkeit des Nomogramms vor allem durch die Entwicklung anhand retrospektiver Daten - abzulesen an dem weiten $95 \%$-Konfidenzintervall für das Gesamtüberleben, so die Forscher.

Fazit: Mithilfe eines neu entwickelten Nomogramms lassen sich das Risiko für Metastasenbildung sowie das Gesamtüberleben von kurativ behandelten Osteosarkompatienten innerhalb von 3 und 5 Jahren relativ gut vorhersagen.

Barbara Kreutzkamp

Ogura K et al. Development and external validation of nomograms predicting distant metastases and overall survival after neoadjuvant chemotherapy and surgery for patients with nonmetastatic osteosarcoma: A multi-institutional study. Cancer. 2015;121(21):3844-52.

\title{
Rhabdomyosarkom: Tumorgröße ist Risikofaktor
}

\section{Häufigster Grund für ein Therapieversagen nach kurativer Therapie eines Rhabdomyosarkoms sind Lokalrezidive. In einer Studie wurde nach Faktoren gesucht, um die Lokalkontrolle gefährdeter Kinder durch angepasste Therapien zu verbessern.}

\footnotetext{
— twa zwei Drittel der Kinder mit einem - Intermediärrisiko-Rhabdomyosarkom (RMS) werden geheilt. Um die Rate weiter zu steigern, sind belastbare Daten zu Risikofaktoren nötig. Zur Identifizierung solcher Faktoren griffen US-amerikanische Wissenschaftler auf die Daten einer Phase-II-Studie der Children's Oncology Group (COG) zurück, in der 702 Kinder mit Intermediärrisiko-RMS behandelt worden waren. Ausgewertet wurden Daten von 423 Patienten im medianen Alter von fünf Jahren. 280 hatten ein embryonales RMS der klinischen Gruppe III, 102 ein alveoläres RMS der Gruppe III und 41 ein alveoläres RMS der Gruppen I-II. Bei Patienten der klinischen Gruppe I-II wurde der Tumor
}

primär operiert, in Gruppe III erfolgte die Exzision in Woche 12. Für die 42-wöchige Chemotherapie wurden Vincristin, Dactinomycin, Cyclophosphamid und Topotecan (VAC oder VAC/VTC) eingesetzt, bestrahlt wurde mit 40-50 Gy.

Nach median 6,6 Jahren lag bei Patienten mit alveolärem RMS der Gruppe I/II die Rate für rückfallfreies 5-Jahresüberleben bei $69 \%$, bei $10 \%$ wurde ein lokaler Progress diagnostiziert. Bei den übrigen Kindern mit einem Gruppe-IIITumor betrugen die Zahlen $70 \%$ bzw. $19 \%$. Histologie, Nodalstatus und Ort der Primärläsion hatten keinen signifikanten Einfluss auf die Rückfallrate, allerdings ergab sich ein Trend für eine erhöhte Lokalrezidivrate bei retroperito- nealen (RP) Tumoren. Eine signifikante Assoziation zeigte sich dagegen bei der Tumorgröße: Tumoren $\geq 5 \mathrm{~cm}$ rezidivierten häufiger als kleinere Tumoren ( 25 vs. $10 \%$; $p=0,0004$ ). Bei Auswertung der Subgruppe mit großen Tumoren zeigte sich, dass praktisch alle RP-Tumoren $5 \mathrm{~cm}$ oder größer waren.

Aufgrund des guten Abschneidens der Kinder mit Gruppe I/II-RMS schlagen die Forscher hier eine mögliche Reduktion der Strahlendosis vor. Bei Kindern mit großen Tumoren könnten, wenn möglich, eine noch spätere Operation sowie verbesserte Bestrahlungsregime die Rezidivrate senken.

Fazit: Das Risiko für Lokalrezidive ist erhöht bei Kindern mit einem RMS der klinischen Klasse III, einer Tumorgröße $\geq 5 \mathrm{~cm}$ sowie tendenziell bei retroperitonealer Lokalisation. Barbara Kreutzkamp

Wolden SL et al. Local control for intermediate-risk rhabdomyosarcoma: results from D9803 according to histology, group, site and size: a report from the Children's Oncology Group. Int J Radiat Oncol Biol Phys. 2015;93(5):1071-6. 